\title{
Scanning Polarization Force Microscopy A Technique for Studies of Wetting Phenomena at the Nanometer Scale
}

\author{
M. Salmeron ${ }^{1}$ \\ 1 Materials Sciences Division, Lawrence Berkeley National Laboratory, \\ University of California, Berkeley, California 94720 - USA \\ e-mail: salmeron@stm.lbl.gov
}

\begin{abstract}
Résumé - Microscope atomique à polarisation : une technique pour étudier les phénomènes de mouillage à l'échelle nanométrique - Nous décrivons une technique, développée récemment et basée sur la mesure de forces électrostatiques à l'aide d'un microscope à force atomique, pour étudier les films liquides et les gouttes. Cette technique, dénommée microscopie atomique à polarisation, est une véritable technique sans contact où la pointe du microscope parcourt la surface à une distance de quelques centaines d'angströms, ce qui est suffisant pour minimiser les perturbations du film liquide. La résolution est de l'ordre d'une centaine d'angströms horizontalement et d'un angström verticalement. L'information fournie par ce microscope comprend la topographie, le potentiel de contact et une cartographie diélectrique de l'échantillon (fonction de la fréquence utilisée).

Après une brève description des concepts de base du mouillage, nous décrivons cette nouvelle technique et illustrons, à travers quelques exemples, quelques-unes de ses applications potentielles.

Mots-clés : mouillage, pression de disjonction, microscope atomique, propriétés diélectriques.
\end{abstract}

\begin{abstract}
Scanning Polarization Force Microscopy: A Technique for Studies of Wetting Phenomena at the Nanometer Scale - We describe a technique developed recently based on the use of electrostatic forces in the atomic force microscope (AFM) to image liquid films and droplets. The technique, named scanning polarization force microscopy (SPFM), is a truly non-contact technique where the tip scans the surface at a height of a few hundred angstroms, which is sufficient to minimize perturbation of the liquid film. The resolution of the technique is a few hundred angstroms horizontally and one angstrom vertically. The information provided by SPFM includes topography, contact potential, and dielectric (frequency dependent) mapping.

After a brief introduction to basic wetting concepts, we describe the technique and illustrate with several examples its potential applications.

Keywords: wetting, disjoining pressure, atomic force microscopy, dielectric properties.
\end{abstract}




\section{INTRODUCTION}

The technique of scanning polarization force microscopy (SPFM) that we will shortly describe provides imaging of liquid structures (films, droplets) with unprecedented resolution in the nanometer range. For that reason, the classic concepts of contact angle and droplet shape, particularly at the edges, require modification. These modifications are due to the surface forces associated with van der Waals, double layer, structural and other forces that have a typical range of a few hundred or perhaps even thousand angstroms. They are not important in the macroscopic range but become dominant within the range in which SPFM operates. Therefore, before describing the technique, we will review the classical wetting formulas and their modification.

When liquid drops form on a flat substrate, they adopt spherical cap shapes (neglecting gravity effects) with a contact angle $\theta$. When the drop dimensions are larger than a few micrometers, this angle depends solely on the interfacial energies, as described by Young's equation:

$$
\gamma_{L V} \cos \theta=\gamma_{S V}-\gamma_{S L}
$$

where $L, V$, and $S$ denote liquid, vapor and solid, respectively. Usually $\gamma_{L V}$ is written without indices simply as $\gamma$. The wetting properties of the surface/liquid system are described by means of the so-called spreading coefficient, defined as:

$$
S=\gamma_{S V}-\gamma_{S L}-\gamma
$$

For a non-volatile liquid, where transport through the gas phase is negligible, one could substitute the first term in the equation $\gamma_{S V}$ with $\gamma_{S 0}$, which corresponds to the interface energy between the dry solid and vacuum. If $S>0$, the liquid will wet the surface, and if $S<0$, a finite contact angle will exist, determined by Equation (1).

When the dimensions of the liquid are below approximately one micrometer, the above relations require modification. If the atomic or molecular interaction potentials are known, the surface energy for very thin films can be calculated by assuming additivity and integrating over the finite thickness of the film below the surface, plus that of the semi-infinite substrate underneath. The value of the energy per unit area obtained in this manner will differ from that of the bulk liquid $\gamma$, by the thickness dependent term $P(e)$. For a non-volatile liquid, the free energy $F$ (per unit area) will then be expressed as:

$$
F(e)=\gamma_{S L}+\gamma+P(e)
$$

A quantity called disjoining pressure $\Pi$ was introduced by Derjaguin et al. (1987) as the natural canonical conjugate of the film thickness, $e$ :

$$
\Pi(e)=-\partial F / \partial e=-\partial P / \partial e
$$

or, equivalently:

$$
P(e)=\int_{e}^{\infty} \Pi(x) \mathrm{d} x
$$

The interaction potentials determining $P(e)$ include van der Waals; electrostatic and entropic double layer forces; hydrophobic forces, arising from the tendency of water to form H-bonded networks; and short-range covalent forces that determine chemisorption and surface reactivity. Except for the van der Waals forces, many of these interactions decay exponentially with distance, giving rise to a disjoining pressure of the form:

$$
\begin{gathered}
\Pi(z)=C \exp \left(-2 \kappa_{D} z\right) \\
\left(\kappa_{D} z>1\right)
\end{gathered}
$$

where $C$ is a constant, $z$ is the distance to the surface and $\kappa_{D}^{-1}$ is the decay length (for example, the screening or Debye length of ions in solutions).

Short-range oscillatory forces arise when the liquid molecules feel the presence of walls of the substrate and form a layered structure near the interface. These forces are also called structural or solvation forces (Israelachvili, 1985).

Other forces can arise as a result of elastic strain on the growing film, which can be due to a surface-induced ordering in the first few layers that reverts to the bulk liquid structure at larger distances. This elastic energy is stored in intermolecular distances and orientations that are stretched or compressed from the bulk values by the influence of the substrate at short distances (Valignat et al., 1996). Similar phenomena are well known to occur in the growth of epitaxial layers in metals and semiconductors.

The shape of a droplet can be determined from the surface energies and interaction forces between the interfaces. These also determine the equilibrium thickness of a liquid film that completely wets a surface. The calculation is done by minimization of the free energy of the total system. The free energy of a droplet with axial symmetry can be expressed as (de Gennes, 1985):

$$
F(z)=F_{0}+\int 2 \pi x \mathrm{~d} x\left[-S+\frac{\gamma}{2}\left(\frac{\mathrm{d} z}{\mathrm{~d} x}\right)^{2}+P(z)+g(z)\right]
$$

where $x$ is the radial distance and the second term comes from the expansion of the length element, assuming that $\mathrm{d} z / \mathrm{d} x$ is small, and $g(z)$ describes the gravitational effects. Supersaturation effects can be introduced by adding a term of the form $\Delta \mu \cdot z$, where $\Delta \mu$ is the difference in chemical potentials of the liquid and vapor phases.

For symmetric droplets, Equation (6) can be integrated by the method of Lagrange multipliers. One interesting case occurs when the potential $P(z)$ is small compared with the surface energy of the liquid and the droplet shape is very 
close to a spherical cap. If the height $e$ and the radius of curvature $R$ at the top of the droplets can be measured, an effective contact angle can be defined through the expression:

$$
\theta^{2}=\frac{2 e}{R}
$$

From Equation (6), the following relation between the effective contact angle and the disjoining pressure under constant volume can be obtained:

$$
\theta^{2}=\theta_{0}^{2}+\frac{2}{\gamma} \cdot[P(e)+e \cdot \Pi(e)]
$$

where $\theta_{0}$ is the macroscopic contact angle. It is clear that the effective contact angle is influenced by the disjoining pressure. We shall use this expression to determine $P(e)$ from experiments with nanoscopic size droplets $(\mathrm{Xu}$ and Salmeron, 1998a).

\section{EXPERIMENTAL STUDIES OF WETTING PHENOMENA}

Many experimental methods have been developed to study wetting phenomena. With an optical microscope, the contact angle of droplets larger than a few micrometers can be measured and the spreading of liquids can be followed. However, to study the effects of the disjoining pressure, one needs films and droplets of sub-micrometer dimensions and therefore instruments with nanometer resolution. Several techniques possessing such resolution in the direction perpendicular to the surface exist today and are used to study the effects of the disjoining pressure. These include the surface force apparatus (SFA) (Israelachvili and Adams, 1976; Israelachvili et al., 1979), ellipsometry, and adsorption isotherm techniques (Schick, 1990). Step-like adsorption isotherms (Cazabat et al., 1990) and layered spreading (Villette et al., 1997a) have been observed. The thickness of these layers is comparable to the molecular diameter, indicating that the disjoining pressure is oscillatory (Villette et al., 1997a).

In recent years, high-resolution X-ray diffraction has become a powerful method for studying layered structures, films, interfaces and surfaces. X-ray reflectivity involves the measurement of the angular dependence of the intensity of the X-ray beam reflected by planar interfaces. If there are multiple interfaces, interference between the reflected X-rays at the interfaces produces a series of minima and maxima, which allows determination of the thickness of the film. More detailed information about the film can be obtained by fitting the reflectivity curve to a model of the electron density profile. Usually, X-ray reflectivity scans are performed with a synchrotron light source. As with ellipsometry, X-ray reflectivity provides good vertical resolution (Daillant et al.,
1988; Bardon et al., 1999) but poor lateral resolution, which is limited by the size of the probing beam, usually several tens of micrometers.

Understanding wetting at smaller lateral scales is important in many applications, such as in corrosion, which occurs at localized regions of the surface. As a result, there is a need for techniques that allow the study of liquid droplets or films with nanometer resolution. A less fundamental but very practical advantage of high lateral resolution is that it opens the way for studies of materials that can not be prepared as very flat samples over large areas. This need has recently been fulfilled, thanks to the explosive development of scanning probe microscopy (SPM) techniques, and in particular, the atomic force microscope (AFM). The AFM has been used to study oscillatory hydration forces between tips and $\mathrm{CaCO}_{3}$ and $\mathrm{BaSO}_{4}$ substrates in water (Cleveland et al., 1995). Tapping-mode AFM has also been used in the imaging of liquid droplets and films (Pompe et al., 1998, 1999; Sheiko et al., 1996; Bardon et al., 1998). In these AFM studies, however, the tip enters in contact with the surface, which makes it difficult to avoid a strong perturbation of the liquid by the imaging tip.

So while imaging of solids or rigid substrates has been revolutionized by SPM, the study of liquids had to avoid contact. Many non-contact SPM methods involve an oscillation of the lever in or out of mechanical resonance. This, however, brings the tip too close to the liquid surface to ensure a truly non-perturbative imaging, at least for lowviscosity liquids. A simple technique developed in 1994 in the author's laboratory not only solves most of these problems but, in addition, provides new information about surface properties. It has been named scanning polarization force microscopy (SPFM) (Hu et al., 1995a, 1995b, 1995c). SPFM not only provides the topographic structure, but also allows the study of local dielectric properties and even the molecular orientation of the liquid.

\section{PRINCIPLE OF OPERATION}

SPFM uses the same technology as the AFM, which is based on sensing the force between sharp tips and the surface, the use of piezoelectric scanners and feedback control electronics. In the AFM, a sharp tip (with typical radii between 100 to $1000 \AA$ ) is used to "feel" by contact the topography of the surface. The tip is mounted on a flexible cantilever with a spring constant on the order of $1 \mathrm{~N} / \mathrm{m}$. The images are obtained by adjusting the separation between the sample and the cantilever such that its bending stays constant while the tip is scanned over the surface. The cantilever bending is usually sensed by the angular deflection experienced by a beam of light reflected off its backside. Due to the load applied in the standard contact operation mode, elastic deformations produce a finite tip-surface contact 
region. The diameter of this region determines the resolution achievable in this AFM mode. One must take into account that due to attractive, capillary and adhesive forces, it is difficult to apply arbitrarily small forces, with the goal of achieving high resolution. The force is typically of a few tens of nanonewtons $(\mathrm{nN})$, which for typical tips and sample materials produces nanometer size contact diameters.

In the SPFM mode, a bias voltage (DC, direct current, or $\mathrm{AC}$, alternating current) is applied to the conductive cantilever $\left(\mathrm{Si}_{3} \mathrm{~N}_{4}\right.$ levers and other insulating levers can be made conductive by coating them with a thin metal film). The opposite charges generated at the tip and surface cause their mutual attraction and this bends the lever toward the surface. Because electrostatic forces are long-range, they provide a means of imaging at a distance that is large enough to minimize perturbation of the liquid surface. The concept of SPFM is schematically shown in Figure 1 (Salmeron et al., 1997). The term polarization force stresses the physical origin of the interactions that give rise to contrast in the images and can be exploited by performing dielectric spectroscopy. Typically, tip-sample operating distances are of a few hundred angstroms when the applied bias is on the order

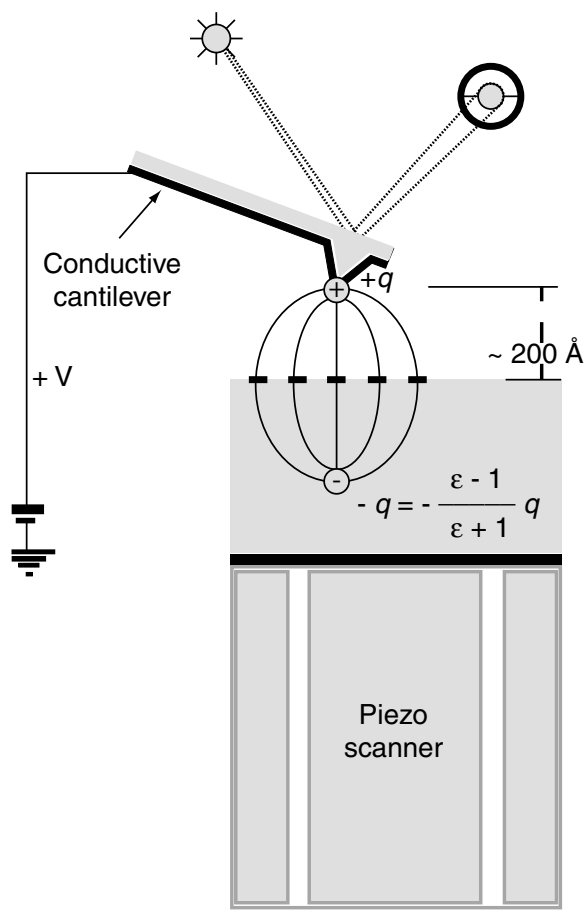

Figure 1

Schematic representation of the operation of the SPFM. An electrically biased AFM tip is attracted towards the surface of any dielectric material. The polarization force depends on the local dielectric properties of the substrate. SPFM images are typically acquired with the tip scanning at a height of 100 to $300 \AA$ (from Salmeron et al., 1997). of a few volts. This produces an attractive force in the nanonewton range. The advantage of the long-range non-contact SPFM operation comes at the price of diminished lateral resolution compared to the contact mode. This resolution is on the same order as the tip-surface separation, i.e., a few hundred angstroms. This is still far better than the typical optical resolution of several micrometers. The vertical resolution of the SPFM, on the other hand, is as good as that of other SPM probes, i.e., in the angstrom region, as determined by the noise of the apparatus.

\subsection{Dependence of the Electrostatic Force on Distance}

From electrostatic arguments it is clear that the force can be written in the form (Martin et al., 1988):

$$
\begin{aligned}
F & =-\frac{1}{2} \cdot \frac{\partial C}{\partial z} \cdot\left(V_{\text {tip }}-\phi\right)^{2} \\
& \approx-4 \pi \varepsilon_{0} \cdot \frac{\varepsilon-1}{\varepsilon+1} \cdot f\left(\frac{R}{z}\right) \cdot\left(V_{\text {tip }}-\phi\right)^{2}
\end{aligned}
$$

where $C$ is the system capacitance, $z$ the tip-sample distance, $\varepsilon$ the local dielectric constant and $R$ the tip radius. $V_{\text {tip }}$ is the applied bias and $\phi$ is the local contact potential difference so that $V_{\text {tip }}-\phi$ represents the total voltage drop. During imaging, the attractive force (lever deflection) is kept constant by the feedback control electronics, and a constant force image is obtained. In DC mode images, dielectric constant and surface potential contributions are mixed with topographic information. The function $f(R / z)$ depends on the geometry of the tip and sample. For a point charge on a flat surface, $f$ is of the form $1 / z^{2}$, but for a real tip the dependence is more complicated. Although an analytical expression for the function $f(R / z)$ can not be obtained even for simple tip shapes (Hao et al., 1991; Belaidi et al., 1998), the following approximation is useful in many cases. The tip and lever can be approximated by a sphere of radius $R$ (the tip apex) separated by a distance $z$ from the surface, and a flat parallel condenser plate at a distance $z+D$, representing the lever. This is shown schematically in Figures $2 \mathrm{a}$ and $2 \mathrm{~b}$.

These two elements are treated as two capacitors in parallel. The capacity of a sphere relative to the sample can be calculated exactly by the method of images. An approximate but analytical expression for the capacitance can be found by integrating the contributions of flat infinitesimal rings of spherical surface centered at the apex (Frantz et al., 1996). The result is:

$$
C(z) \approx 2 \pi \varepsilon_{0} R \times\left[\ln \left(\frac{R}{z}\right)+a\right]
$$

where $a \approx 2$.

The capacitance of the supporting lever is estimated to be that of a flat plate with area $S$ at a distance $z+D$ from the 

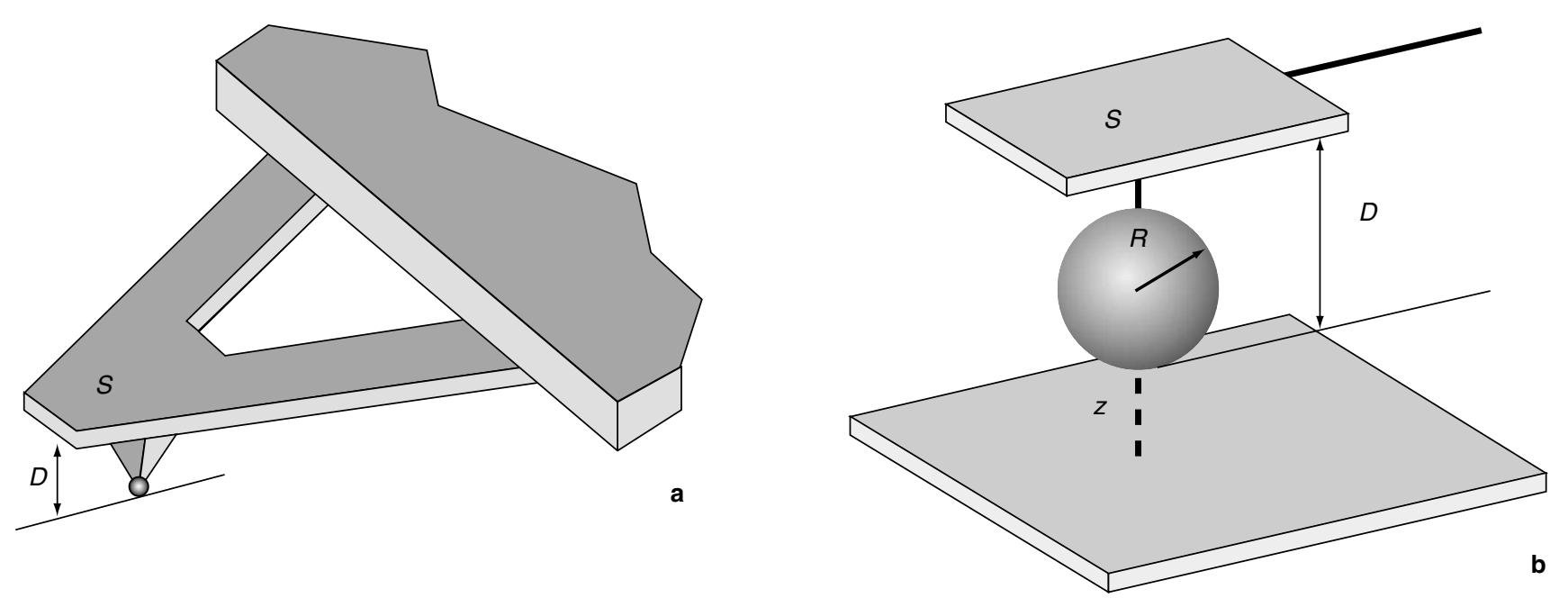

Figure 2

Model for the calculation of electrostatic forces. The tip-lever system (a) is approximated by a sphere of radius equal to the apex tip radius $R$, and flat plate of area $S$ equal to that of the support lever (b).

surface. Its contribution is $C_{\text {lever }} \approx \varepsilon_{0} S /(z+D)$, and the force due to the lever is $F_{\text {lever }} \propto-\partial C_{\text {lever }} / \partial z \approx C_{\text {lever }} /(z+D)$. An estimation of the orders of magnitude involved is useful. For $R=1000 \AA$ and for $z=R / 10$ (i.e., for $z=100 \AA$ ), we get $C_{\text {tip }} \approx 20 \mathrm{aF}$, while using $S=25 \times 50 \mu \mathrm{m}^{2}$ (the moving part of the lever) and $D=4 \mu \mathrm{m}$ for the lever we get $C_{\text {lever }}=2.7 \mathrm{fF}$. So it is clear that, at typical operating distances, the capacitance is dominated by the lever (by a factor $\sim 100$ ) and not by the tip. An actual measurement of the capacitance would yield an even higher value $(\mathrm{pF})$ because it would include the nonmoving part of the lever and its support chip. The force, however, is comparable, with $\partial C_{\text {lever }} / \partial z \approx \partial C_{\text {tip }} / \partial z$ at $z=R / 10$. Moreover, while $\partial C_{\text {lever }} / \partial z$ stays constant during imaging (because $z<<D), \partial C_{\text {tip }} / \partial z$ contains all the topographic information. So we conclude that, at typical imaging distances $(\sim 100 \AA)$, and for typical tips used in AFM, the capacitance is due to the whole lever while the varying part of the force is due to the tip apex. This is, of course, why high-resolution imaging is at all possible in SPFM.

The sphere-plane capacitor model gives a useful approximate expression for the function $f(R / z)$. In the region $0<z / R<1$, which is typical in SPFM imaging, $f$ can be approximated by a $1 / z$ dependence $(E q .(10))$. The planar lever adds a nearly constant term. Thus for the range $0<z<$ $R$, we have the following approximate function for the electrostatic force:

$$
F \approx\left(\frac{A \cdot R}{z}+B\right) \cdot(V-\phi)^{2} \mathrm{~N} \cdot \mathrm{V}^{-2}
$$

The constants $A$ and $B$ are both on the order of $10^{-11}$. This simple relation is indeed found experimentally. For larger values of $z / R$, the functional form of $f$ deviates from $1 / z$.
The fact that the dielectric constant depends on the frequency ( $\mathrm{Xu}$ and Salmeron, 1998a) gives SPFM an interesting spectroscopic character. Local dielectric spectroscopy, i.e., the study of $\varepsilon(\omega)$, can be performed by varying the frequency of the applied bias. Application of this capability in the RF range has been pursued by Xiao et al. in the study of metal and superconductor films (Wei et al., 1996; Takeuchi et al., 1997) and dielectric materials (Gao and Xiang, 1998). In these applications, a metallic tip in contact with the surface was used.

Rotational spectroscopy of polar molecules is a promising potential of the technique that is worth pursuing. Rotational modes, however, occur at extremely high frequency, in the $\mathrm{GHz}$ range for free molecules. This puts severe restrictions in the shape and shielding of the tips and wiring. Wave-guide type designs for the microfabricated tips are necessary. Their feasibility was demonstrated by van der Weide (1997). For the present status of tips available today, only experiments in the low-frequency range of the spectrum can be performed routinely. We will present examples of the exploitation of this capability in studies of ionic mobility in the sections below.

\subsection{Separation of Topography and Contact Potential}

As we have shown, the polarization force depends not only on the topography (through the $f(R / z)$ term) and dielectric constant $\varepsilon$, but also on the local contact potential $\phi$. As we shall see now, AC bias modulation and lock-in detection allow these contributions to be separated.

Experiments using AC bias modulation for the purpose of separating topography and contact potential were first carried out by Schönenberger and Alvarado (1990) and later by 
Yokoyama et al. (1993). When the cantilever is driven by a voltage of frequency $\omega$, the force detected by the lever can be expressed as:

$$
\begin{aligned}
F & =B \cdot f\left(\frac{R}{z}\right)\left(V_{\text {tip }} \sin (\omega t)-\phi\right)^{2} \\
& =B \cdot f\left(\frac{R}{z}\right) \\
& {\left[-\frac{1}{2} V_{\text {tip }}^{2} \cos (2 \omega t)-2 V_{\text {tip }} \phi \sin (\omega t)+\frac{1}{2} V_{\text {tip }}^{2}+\phi^{2}\right] } \\
& =F(2 \omega)+F(1 \omega)+F(0 \omega)
\end{aligned}
$$

where:

$$
B=-4 \pi \varepsilon_{0} \cdot \frac{\varepsilon-1}{\varepsilon+1}
$$

The amplitudes of the signals with frequencies $2 \omega$ and $1 \omega$ can be easily measured using lock-in amplifiers. The $2 \omega$ signal is used as control signal in the feedback electronics so that its value is kept constant. The feedback output is then the topographic image (modulated by $\varepsilon(x, y)$ ). Since the term $B \cdot f(R / z)$ in Equation (12) is kept constant by the feedback, the $1 \omega$ signal obtained simultaneously with another lock-in amplifier provides a map of surface charge or surface potential distribution. Alternatively, one can add a DC offset to null the $F(1 \omega)$ signal, as in the Kelvin method for work function measurements. Names such as scanning Kelvin probe microscopy (Henning et al., 1995) and scanning surface potential microscopy (Fujihira, 1999) have been used for the technique. As we shall see, the surface potential can provide important information on the charge distribution or dipole orientation.

\section{APPLICATIONS}

\subsection{Disjoining Pressure Effects on the Contact Angle of Small Droplets}

Ideally one would like to visualize the molecular scale details at the edge of a droplet to obtain direct information about the molecular nature of wetting. This is not always possible, particularly when these details have dimensions below $\sim 300 \AA$, the resolution limit of SPFM. However, the height and curvature of a droplet can usually be measured accurately. These parameters can then be used to obtain an effective contact angle. We present here a few examples of this type of study.

Droplets of various liquids were prepared in several ways. For example, a macroscopic drop was first deposited on the substrate and then absorbed from an edge using filter paper. In other cases, a macroscopic drop was blown away with a jet of $\mathrm{N}_{2}$ or air. These processes leave a surface that appears dry to the naked eye but still contains many tiny droplets that can be observed with SPFM. If the droplets are of aqueous solutions, the water vapor pressure in the chamber, with which they readily equilibrate, determines their size. For liquids with low vapor pressure, films and droplets can be formed by condensation from a warmed reservoir.

Figure $3 \mathrm{a}$ shows $\mathrm{H}_{2} \mathrm{SO}_{4}$ droplets on mica at very low humidity $(<5 \%)$. The contact line around the drops is smooth and circular, revealing that no pinning has occurred (Rieutord and Salmeron, 1998). Although the area between drops is flat, it does not correspond to clean mica but to a liquid film covering it of a few monolayers thickness. This is deduced from the hysteresis in the force $v s$. distance

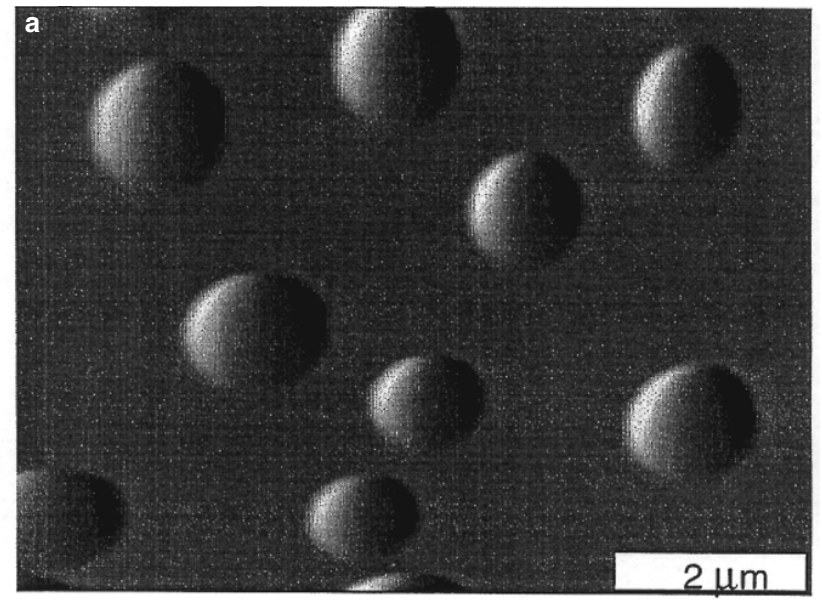

Figure 3

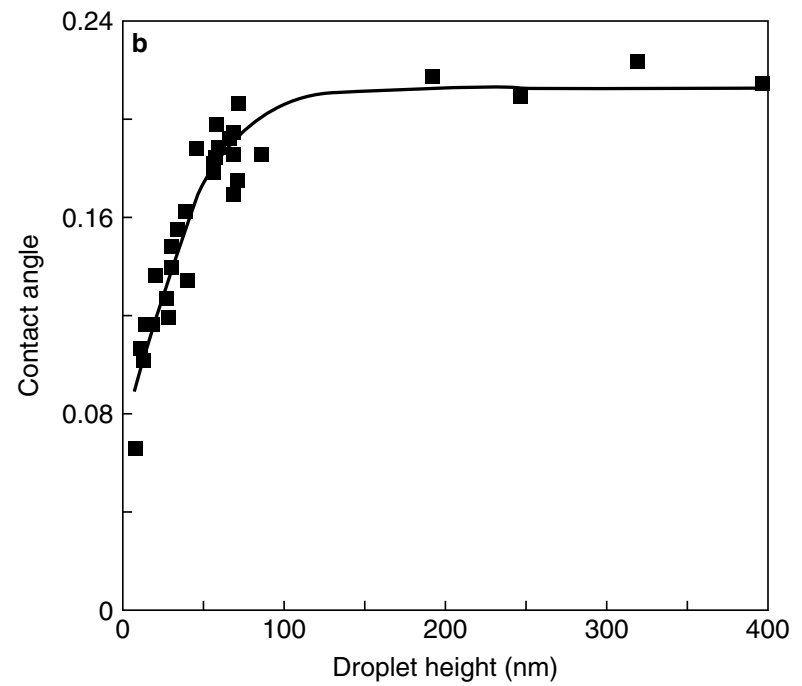

(a) SPFM image showing $\mathrm{H}_{2} \mathrm{SO}_{4}$ droplets deposited on mica at low humidity $(<5 \%)$. The region between drops is covered by a thin acid film. (b) The effective contact angle of $\mathrm{H}_{2} \mathrm{SO}_{4}$ on mica measured by the relation $\theta^{2}=2 e / R(e=$ droplet height; $R=$ curvature radius at the top) as a function of droplet height. Solid line shows fit with disjoining pressure given by Equation (8). 
experiments, where the tip is brought into contact with the surface and then pulled off.

The observation of liquid drops on top of a film of the same substance is often referred to as pseudo-partial (Brochard-Wyart et al., 1991) or autophobic wetting. This behavior is not surprising in this case due to the large surface energy of cleaved mica. Values of $300-600 \mathrm{~mJ} / \mathrm{m}^{2}$ have been reported for the surface energy of freshly cleaved mica (Sheiko et al., 1997), compared with $53 \mathrm{~mJ} / \mathrm{m}^{2}$ for the surface tension of sulfuric acid.

When plotted as a function of drop size (Fig. 3b), the contact angle was found to decrease with increasing drop height. Using Equations (7) and (8), the interaction potential can be described with an exponential function of the form:

$$
P(z)=-1.4 \exp (-z / 250 \AA) \mathrm{mJ} / \mathrm{m}^{2}
$$

\subsection{Glycerol}

Glycerol is well-known for its strong H-bonding in the liquid phase and its high viscosity. To study its wetting properties, we deposited glycerol on mica by condensation from its vapor in standard laboratory air (Xu et al., 1998). On freshly cleaved mica, condensation begins with the formation of ellipsoidal droplets. These droplets grow laterally and become pancakes with a relative contrast or height of $30 \AA$. The pancakes spread until the film completely covers the surface.

A very different behavior is observed when condensation occurs on a mica surface that has been exposed to air for a few hours (we will refer to it as contaminated mica). In this case, glycerol forms droplets in the shape of spherical caps, indicating that it does not completely wet the surface. This behavior is similar to that of water. The contact angle of water on mica surfaces increased from $0^{\circ}$ on the freshly cleaved surface to a small value between $2^{\circ}$ and $3^{\circ}$ on the contaminated mica (Xu et al., 1998).

On the contaminated and slightly hydrophobic surface, the spherical droplets grow continuously with time, as shown in the sequence of images in Figure 4a. When two droplets grow close enough to touch each other, they coalesce, as can be seen in several images in the figure.

The effective contact angle values $(\theta)$ measured as a function of drop height are shown in the graph of Figure $4 \mathrm{~b}$. A rapid decrease of $\theta$ is observed when the height falls below $200 \AA$. This indicates that the potential $P(z)$ is negative. An exponential dependence of the disjoining pressure with distance gives a very good fit, as shown in the inset of Figure 4b, which shows a semilog plot of $P(z)-z P^{\prime}(z) v s . z$. As in the case of sulfuric acid, we find that a pure exponential function:

$$
P(z)=-6.4 \times 10^{-5} \exp (-z / 50 \AA) \mathrm{J} / \mathrm{m}^{2}
$$

describes the results in a satisfactory way. The exponential dependence is indicative of hydrophobic attractive forces
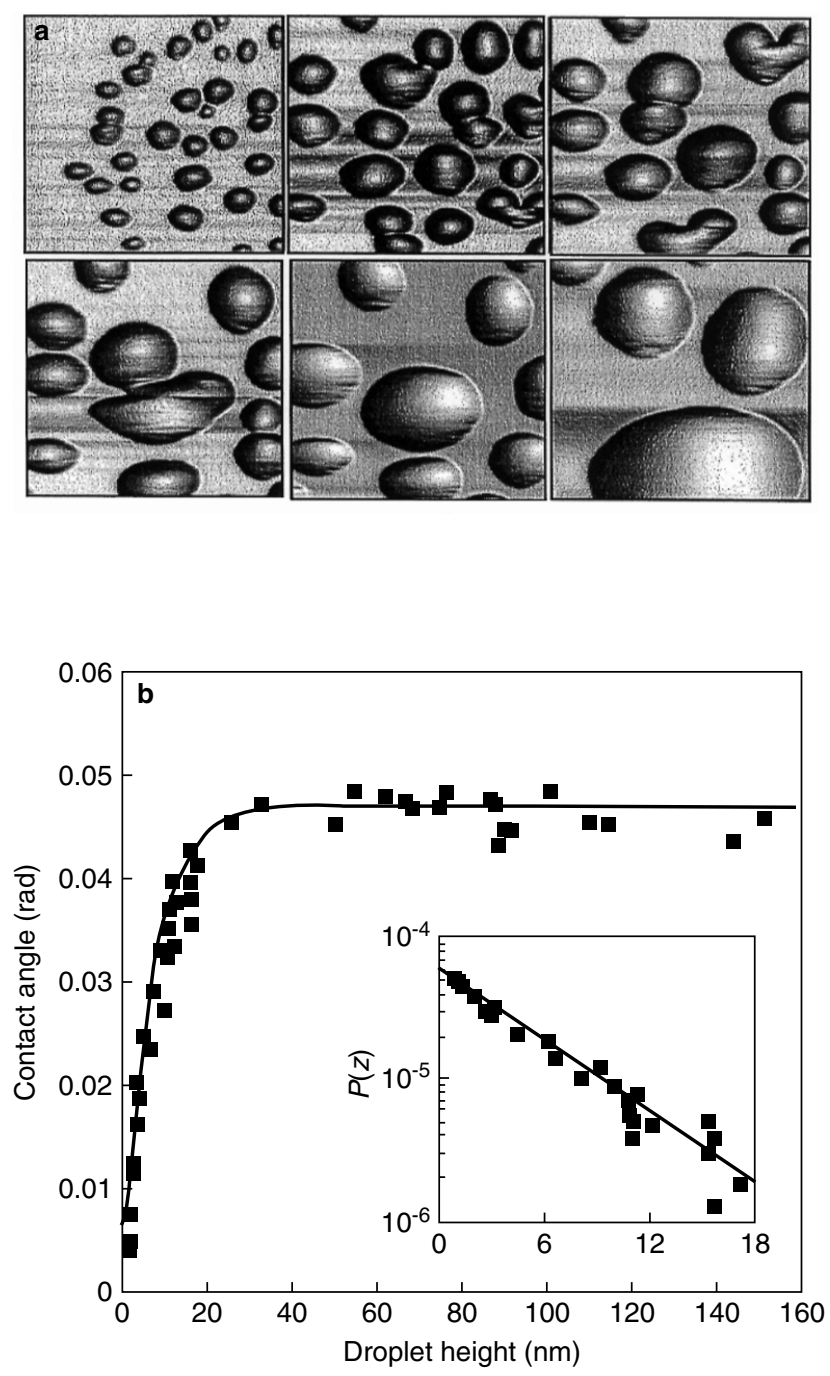

Figure 4

(a) $15 \mu \mathrm{m} \times 15 \mu \mathrm{m}$ SPFM images showing the condensation of glycerol on contaminated mica. Droplets with a nearly spherical cap shape are formed and increase indefinitely in size as condensation continues. When two drops establish contact, they coalesce into a larger drop.

(b) A plot of the effective contact angle $\theta$ of the glycerol drops on contaminated mica $v s$. drop height $e$. A rapid increase occurs up to $200 \AA$ (corresponding to a drop with a base diameter of $\sim 2.2 \mu \mathrm{m})$, where it reaches a constant value $\theta_{0}$. Inset: Plot of $\left(\theta_{0}^{2}-\theta^{2}\right) /(1+e / \delta) v s . e$. This plot shows that the potential due to the long-range forces depends exponentially on the distance (from $\mathrm{Xu}$ and Salmeron, 1998a).

between the glycerol-air interface and the glycerol-mica interface. This potential gives a negative disjoining pressure of $\sim 1 \mathrm{~atm}$ at $z$ close to 0 . The strength of the force appears to be 100 times lower than that typical for water between hydrophobic surfaces (Israelachvili, 1985). 


\subsection{Layering of Liquid Crystals}

In this section, we show an example in which the molecular scale structure of the film is manifested in the form of features that can be resolved with the SPFM. These are steps and terraces that appear at the edge of droplets and films, when the molecules form uniform layers during spreading. This has already been observed with ellipsometry when the terraces are many tens of micrometers wide (Villette et al., 1997b; Valignat et al., 1993). With SPFM, molecular terraces can be observed at much higher resolution, so that we can study at a much finer scale the phenomena of wetting and dewetting. In addition to the topographic images, SPFM can provide simultaneous images of surface contact potential, which provide additional useful information on the structure of molecules, particularly when these are polar.

The wetting and spreading properties of liquid crystals (LC) on solid substrates are of interest due to their use in display devices. In addition, these interesting liquids provide a good testing ground for fundamental theories of wetting. Films of 8CB (4'-n-octyl-4-cyanobiphenyl) have been studied by optical microscopy, X-ray reflectivity (Bardon et al., 1999; Pershan, 1989), ellipsometry (Valignat et al., 1996; Bardon et al., 1999), second-harmonic generation (Feller et al., 1991), scanning tunneling microscopy (STM) (Stevens et al., 1998), AFM (Fang et al., 1997), and SFA (Artsyukhovich et al., 1999).

Bulk 8CB exists in three phases. With increasing temperature, there is a transition at $21.5^{\circ} \mathrm{C}$ from crystalline to the smectic-A phase; at $33.5^{\circ} \mathrm{C}$, there is a second-order transition to a nematic phase; and at $40.5^{\circ} \mathrm{C}$ there is a weak first-order transition to an isotropic liquid (60). In bulk, the 8CB molecules are paired into dimers (1.4 times longer than a single molecule) with anti-parallel dipole moments. In the smectic-A phase, the dimers form layers spaced by $31.7 \AA$, with the dimers aligned perpendicular to the layers (61). Ellipsometry and X-ray reflectivity have been used to study the spreading and structure of macroscopic (in $x, y$ ) films of 8CB on silicon wafers (Bardon et al., 1998, 1999). It was found that a prewetting film is formed during spreading in the smectic and nematic phases. The prewetting film is composed of a monolayer of single $8 \mathrm{CB}$ molecules with a thickness of $8 \AA$, connected to a $41 \AA$ thick film. This film consists of $\mathrm{a} \sim 33 \AA$ thick bilayer on top of the $8 \AA$ monolayer. Both monolayer and trilayer extend over millimeter distances.

For our studies of the wetting properties of $8 \mathrm{CB}$, a small amount of liquid was directly deposited on the substrate $\mathrm{Xu}$ et al., 2000). For studies of the nematic and isotropic phases, $8 \mathrm{CB}$ was condensed from its vapor while keeping the Si substrate at the appropriate temperature (the vapor was produced by heating an $8 \mathrm{CB}$ reservoir to $80^{\circ} \mathrm{C}$ in front of the substrate). When studying the spreading of sub-micrometer
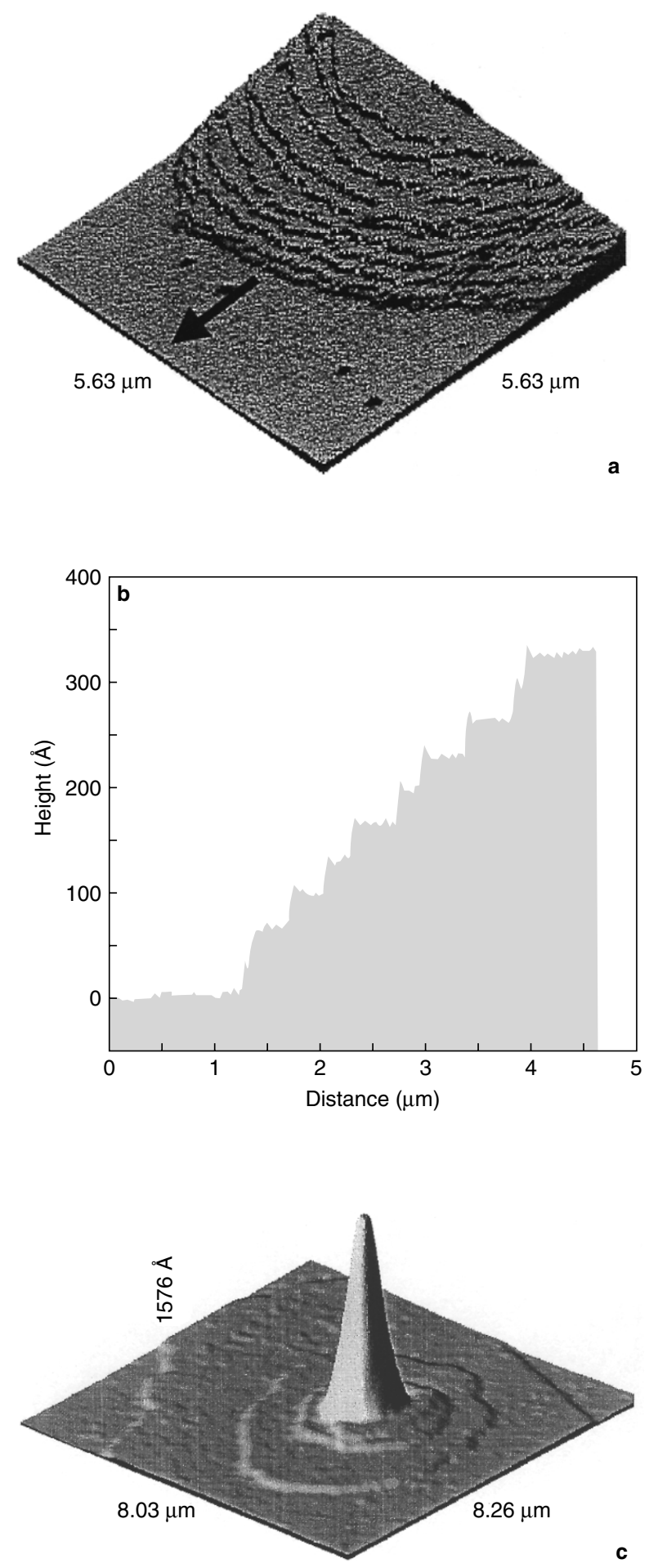

Figure 5

(a) SPFM image of the spreading front of a smectic drop of 8CB liquid crystal on a Si wafer, showing a layered structure. Each layer is $32 \AA$ thick. The layers advance in the direction of the arrow at the rate of $20-30 \AA / \mathrm{s}$ at room temperature.

(b) Profile of the droplet front showing the steps.

(c) Drop and surrounding smectic layers. Vertical scale is greatly exaggerated (from Xu et al., 2000). 
size deposits of $8 \mathrm{CB}$, we observed a behavior similar to that found at macroscopic scales (Bardon et al., 1999): 8CB forms droplets and pancakes on the surface in the smectic phase, wets the surface in the nematic phase, and dewets it in the isotropic phase. However, we found that rapid cooling to room temperature, combined with the high viscosity of $8 \mathrm{CB}$, freezes the liquid in the phase corresponding to the substrate temperature during deposition for days.

Molecular multilayer spreading was observed with SPFM. This is shown in Figure 5a, which was obtained near the edge of a large spreading drop. It shows a layered structure with $32 \AA$ steps (all $z$ values have an associated error bar of $\pm 1 \AA$ ), the thickness of a smectic bilayer. The cross-section in Figure 5b, in the direction of the arrow shown in Figure 5a, shows the perfect layering of the smectic bilayers. Layering near the edge of a spreading droplet has never been observed before with other techniques. In a sequence of time-lapsed images, the layers can be seen advancing at an average speed of 20-30 $\mathrm{A} / \mathrm{s}$ at room temperature. The layers advance on top of the prewetting trilayer, which extends over millimeter distances in front of the droplet. Although the stable state of $8 \mathrm{CB}$ on silicon is in the form of a pancake, we often observed metastable nanometer-scale droplets surrounded by layered structures. Figure $5 \mathrm{c}$ shows an example of this. The droplet is not spherical. A higher resolution image shows that the lower part of the droplet is also layered with steps $32 \AA$ high.

The vapor deposition method at variable substrate temperature provides additional insight into the structure and wetting properties of $8 \mathrm{CB}$ in its various phases. If the substrate temperature is between $41^{\circ}$ and $33^{\circ} \mathrm{C}$, flat islands $32 \AA$ thick are formed if only a small amount of $8 \mathrm{CB}$ is condensed on the surface. The size of these flat islands increases with deposition time while their height remains constant until a uniform layer is formed. If more $8 \mathrm{CB}$ is deposited, droplets form on top of the film.

On samples prepared with a substrate temperature above $41^{\circ} \mathrm{C}$, only spherical droplets were observed. Although no change could be observed in the droplets for several days, we noticed that, by applying a strong attractive electrostatic force, the tip could induce the droplets to spread and form pancakes. This indicates that the droplets are indeed in a metastable state. To determine whether the $8 \mathrm{CB}$ droplets condensed above $41^{\circ} \mathrm{C}$ (trapped in the isotropic phase) sit on a trilayer or on bare silicon, we used the AFM tip to mechanically spread the droplets and thus accelerate their conversion to a stable configuration. The SPFM images shown in Figures $6 \mathrm{a}$ and $6 \mathrm{~b}$ were obtained after such tipinduced spreading. A layered structure with $\sim 32 \AA$ high steps typical of the smectic phase is obtained. The first or bottom layer is $41 \AA$ thick, while the layers above it are all $32 \AA$ thick. This indicates that the bottom layer of the film is a trilayer and that the remaining substrate is dry silicon, i.e., not covered with either a monolayer or trilayer of $8 \mathrm{CB}$. We can thus conclude that the frozen isotropic droplets sit on
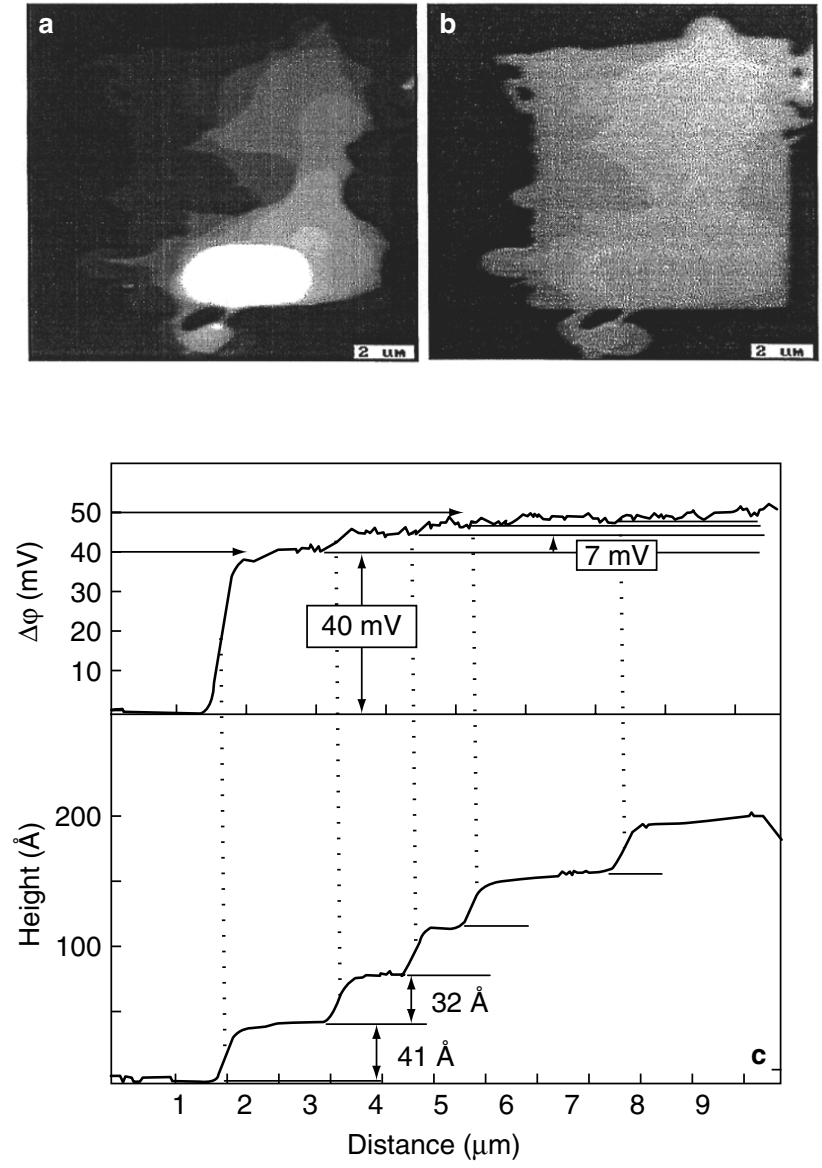

Figure 6

(a) Topographic and (b) contact potential SPFM images obtained after spreading an $8 \mathrm{CB}$ droplet deposited on silicon at a temperature above $41^{\circ} \mathrm{C}$, by contact with the AFM tip scanning over a square area. Several layers can be observed. The first one is $41 \AA$ thick and the following ones are $32 \AA$ thick, as shown in (c). The bright spot near the bottom is the remaining unspread droplet. The potential in the first layer is $40 \mathrm{mV}$ higher than that of the substrate and that of the second layer is $7 \mathrm{mV}$ higher than the first. The third and fourth layer potentials are 2 and $1 \mathrm{mV}$ higher, respectively, as shown in (c). Above that, the increase is negligible (from $\mathrm{Xu}$ et al., 2000).

bare silicon. By contrast, the flat layer formed by evaporation between $33^{\circ}$ and $41^{\circ} \mathrm{C}$, is $32 \AA$ high, indicating that it consists of a bilayer on top of the monolayer. The monolayer must have formed very rapidly during the first stages of exposure to the vapor.

The simultaneously acquired contact potential images provide important structural information on the liquid film. The droplets formed at temperatures $>41^{\circ} \mathrm{C}$ have a surface potential $50 \mathrm{mV}( \pm 3)$ higher than the surrounding background (Fig. 6c). The $32 \AA$ film formed between $41^{\circ}$ and $33^{\circ} \mathrm{C}$ has a potential about $40 \mathrm{mV}$ higher than the surrounding monolayer and the droplets formed above that 
film have a potential $10 \mathrm{mV}$ higher than the bilayer film. In the structure formed by tip-induced spreading, the bottom trilayer has a surface potential $40 \mathrm{mV}$ higher than the surrounding substrate while the bilayer above it is $7 \mathrm{mV}$ more positive. The third bilayer adds $2 \mathrm{mV}$ and the fourth adds $\sim 1 \mathrm{mV}$. Layers above the fourth make negligible contributions. The total potential increase at the top of pancakes or multilayers is $\sim 50 \mathrm{mV}$ higher than that of the bare silicon substrate, which is similar to that of the frozen isotropic droplets. This indicates that the isotropic droplets have similarly oriented bottom layers, and the remaining $8 \mathrm{CB}$ molecules above them do not contribute to the droplet potential.

The information obtained from the simultaneously acquired topographic and surface potential images allows us to propose a model for the structure of $8 \mathrm{CB}$ in various wetting situations. We have seen that the potential of the first bilayer (32 $\AA$ thick) is $40 \mathrm{mV}$ higher than the monolayer it sits on. The same contact potential difference is measured between the trilayer (41 A thick) and the bare silicon (Fig. 6). These two observations indicate that the monolayer bound to silicon does not contribute appreciably to the surface potential. This in turn indicates that the molecular dipole moment is parallel to the surface. Since the dipole originates in the charge distribution between the nitrogen and benzene rings, only this part needs to be parallel to the surface. This is consistent with the $8 \AA$ thickness of the monolayer observed by ellipsometry. The $8 \AA$ is contributed by the alkane group at $\mathrm{a} \sim 30^{\circ}$ with the surface, giving a height of $4 \AA$, plus the 4-5 $\AA$ of the flat-lying biphenyl group.

Since the $8 \mathrm{CB}$ dimers in the bulk have no net dipole moment, we conclude that the $40 \mathrm{mV}$ of the first bilayer on top of the monolayer originates from either a distortion of the 8CB pair that misaligns their dipoles (a few degrees misalignment would be sufficient), or to a tilt of the first monolayer, which would no longer be completely parallel to the surface. This tilt would result in a net vertical component of the dipole.

\subsection{Ion Solvation, Mobility and Exchange}

The frequency dependent spectroscopic capabilities of SPFM are ideally suited for studies of ion solvation and mobility on surfaces. This is because the characteristic time of processes involving ionic motion in liquids ranges from seconds (or more) to fractions of a millisecond. Ions at the surface of materials are natural nucleation sites for adsorbed water. Solvation increases ionic mobility and this is reflected in their response to the electric field around the tip of the SPFM. The schematic drawing in Figure 7 illustrates the situation in which positive ions accumulate under a negatively biased tip. If the polarity is reversed, the positive ions will diffuse away while negative ions will accumulate under the tip. Mass transport of ions takes place over distances of a few tip radii, or a few times the tip-surface distance.

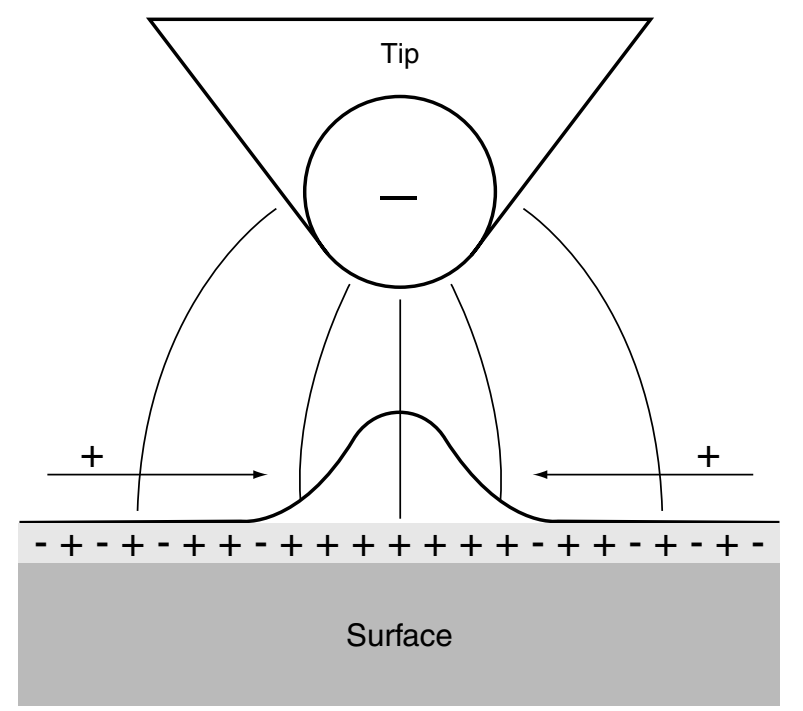

Figure 7

Schematic diagram of a negatively charged tip attracting positively charged ions under it. Diffusion of surface ions is enhanced by solvation in humid environments.

Surface ions are thus expected to substantially contribute to the polarization force at low frequencies. Also, one expects different ions to have different solvation properties and mobility. These phenomena can be studied with SPFM and are important in many processes, including surface reactions, ionic exchange processes between surface and bulk ions, rock weathering, ion sequestration, and other environmental problems.

We have performed ionic mobility studies on mica and in alkali halide surfaces, which we describe now. In humid environments, the ions in the surface of these materials become solvated and mobile. Experiments were performed to determine the response time of the polarization force as a function of humidity. To that effect, a square bias voltage such as the one in Figures $8 \mathrm{a}$ and $8 \mathrm{~b}$ was applied to the tip. The response time of the force to the sudden changes of bias voltage can be easily measured.

In our studies of mica, the $\mathrm{K}$ ions naturally present on its surface were exchanged by other ions by washing it in appropriate solutions. For example, in acids or in pure water, $\mathrm{K}^{+}$is replaced by $\mathrm{H}^{+}$. In $\mathrm{Mg}$ - and Ca-sulfate solutions, $\mathrm{Mg}^{+}$ and $\mathrm{Ca}^{+}$ions replace $\mathrm{K}^{+}$, as verified by $\mathrm{X}$-ray photoelectron spectroscopy (Xu and Salmeron, 1998b). The response time for these different ions as a function of humidity is shown in Figure 9. It is clear that $\mathrm{H}^{+}\left(\right.$or $\left.\mathrm{H}_{3} \mathrm{O}^{+}\right)$ions are much more mobile than $\mathrm{K}^{+}$, while these ions are more mobile than $\mathrm{Mg}^{+}$ and $\mathrm{Ca}^{+}$.

Our studies of water adsorption on alkali halide surfaces (Dai et al., 1997; Luna et al., 1998) can be summarized as follows. For all crystals studied $(\mathrm{NaCl}, \mathrm{KCl}, \mathrm{KBR}$ and $\mathrm{KF}$ ), 

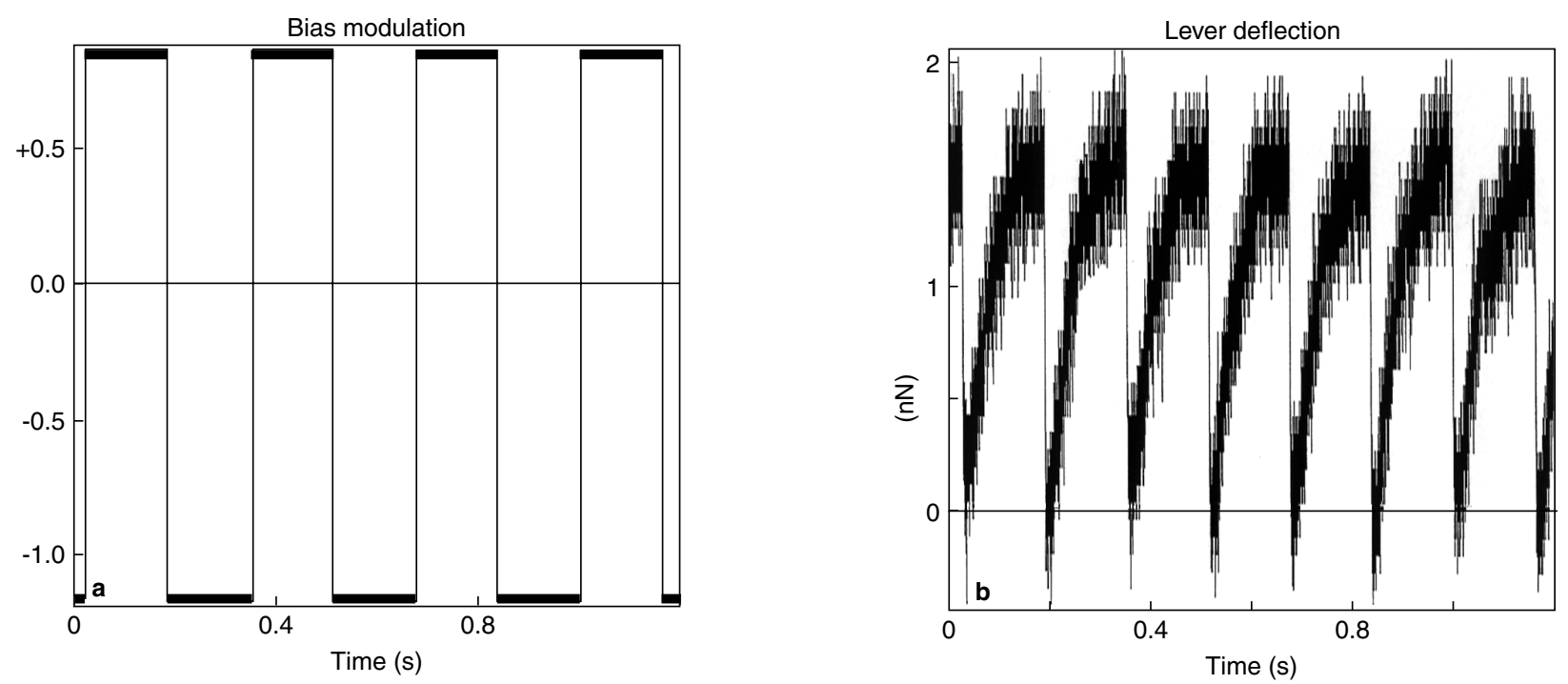

Figure 8

(a) Square voltage waveform applied to the tip.

(b) Corresponding changes in tip deflection (converted to force after multiplying by the lever spring constant).

There is a net attractive force for both the positive and the negative cycles, but it takes time to reach the final force value. Note that the square wave voltage is not symmetric around zero. An offset is applied to compensate for the contact potential difference between the tip and surface. This offset is dependent on humidity and is equal to the potential difference between the tip and sample (from Luna et al., 1998).

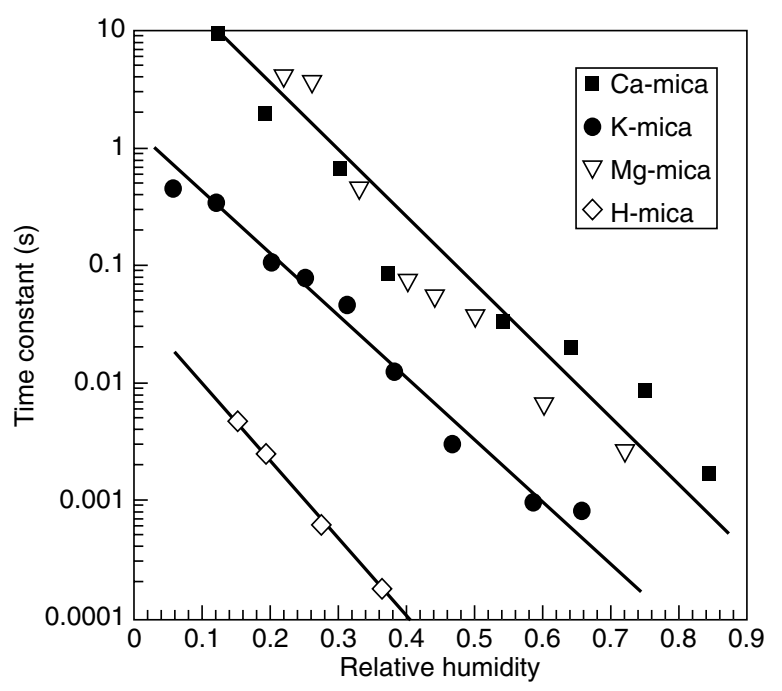

Figure 9

Semi-log plot of the force-response time measured over mica and ion-exchanged mica surfaces as a function of relative humidity. The time constant characterizes the mobility of different surface ions at different humidity values (from Xu and Salmeron, 1998b).

there is a humidity value at which the first water monolayer reaches saturation, a result that agrees with the infrared spectroscopy results of Ewing and Peters (1997). Below this value, water solvates preferentially cations at the step edges.
The mobile cations remain electrostatically bound in the vicinity of the negatively charged steps, giving rise to a decoration ribbon and making the local surface potential positive. The average surface potential also becomes more positive. Above the monolayer, both cations and anions dissolve at similar rates, thus removing the fixed negative charge accumulated at the steps and triggering rapid step motion. The solvation of anions above the monolayer produces a decrease in surface potential and a change in the response time. Rapid displacement of step edges can now take place, since both cations and anions are dissolving, thus making mass transport possible. Finally, upon reaching the deliquescence point, the surface step structure collapses and a thick liquid film is formed.

\section{CONCLUSION}

An AFM-based technique, scanning polarization force microscopy, has been developed that is capable of imaging liquid films and droplets with a resolution of a few hundred angstroms in the $x y$ plane, and one angstrom in $z$. This technique operates on the principle of electrical polarization of the substrate and the ensuing electrostatic forces. It can be operated in the DC and AC modes to provide a wealth of information. Topographic mapping and surface potential mapping can be performed simultaneously. In addition, the frequency of the AC bias can be varied to distinguish between different surface processes that have characteristic 
response times. At present, with frequencies in the range of up to several $\mathrm{kHz}$, SPFM provides a very useful tool to study ionic mobility, particularly on surfaces that can adsorb water from moist environments. In that respect, SPFM should be a powerful tool in environmental research, in the weathering of rocks and other materials that are present in the form of microparticulates in the atmosphere. SPFM has demonstrated its usefulness in studies of disjoining pressure, by measuring the shape of nanometer size droplets on surfaces.

Further developments promise an even brighter future for the technique. We are currently working on extending the frequency to the $\mathrm{MHz}$ range, which might open the way to spectroscopic and dielectric studies of polar molecules. As we have discussed, several successful attempts at implementing high-frequency modulation have already been carried out in several laboratories.

\section{ACKNOWLEDGMENT}

This work was supported by the Director, Office of Science, Office of Basic Energy Sciences, Materials Sciences Division, of the US Department of Energy under contract number DE-AC03-76SF00098.

\section{REFERENCES}

Artsyukhovich, A., Broekman, L.D. and Salmeron, M. (1999) Friction of the Smectic-A Liquid Crystal (8CB) as Probed by Surface Forces Apparatus. Langmuir, 15, 6, 2217-2223.

Bardon, S., Ober, R., Valignat, M.P., Vandenbrouck, F., Cazabat, A.M. and Daillant, J. (1999) Organization of Cyanobiphenyl Liquid Crystal Molecules in Prewetting Films Spreading on Silicon Wafers. Phys. Rev. E, 59, 6, 6808-6818.

Bardon, S., Valignat, M.P., Cazabat, A.M., Stocker, W. and Rabe, J.P. (1998) Study of Liquid Crystal Prewetting Films by Atomic Force Microscopy in Tapping Mode. Langmuir, 14, 10, 2916-2924.

Belaidi, S., Lebon, F., Girard, P., Leveque, G. and Pagano, S. (1998) Finite Element Simulations of the Resolution in Electrostatic Force Microscopy. Appl. Phys. A, 66, 1-2, S239S243.

Brochard-Wyart, F., di Meglio, J.M. and de Gennes, P.G. (1991) Spreading of Nonvolatile Liquids in a Continuum Picture. Langmuir, 7, 335-338.

Cazabat, A.M., Fraysse, N., Heslot, F. and Carles, P. (1990) Spreading at the Microscopic Scale. J. Phys. Chem., 94, 19, $7581-7585$

Cleveland, J.P., Schaffer, T.E. and Hansma, P.K. (1995) Probing Oscillatory Hydration Potentials Using Thermal-Mechanical Noise in an Atomic-Force Microscope. Phys. Rev. B, 52, 12, R8692-8695.

Dai, Q., Hu, J. and Salmeron, M. (1997) Adsorption of Water on $\mathrm{NaCl}$ (100) Surfaces: The Role of Atomic Steps. J. Phys. Chem. $B, \mathbf{1 0 1}, 11,1994-1998$.

Daillant, J., Benattar, J.J., Bosio, L. and Leger, L. (1988) Final Stages of Spreading of Polymer Droplets on Smooth Solid Surfaces. Europhys. Lett., 6, 5, 431-436.
De Gennes, P.G. (1985) Wetting: Statics and Dynamics. Rev. Mod. Phys., 57, 3, 827-863.

Derjaguin, B.V., Churaev, N.V., Muller, V.M. and Kitchener, J.A. (1987) Surface Forces, Consultants Bureau, New York.

Ewing, G.E. and Peters, S.J. (1997) Adsorption of Water on NaCl. Surf. Rev. Lett., 4, 4, 757-770.

Fang, J., Knobler, C.M. and Yokoyama, H. (1997) Layer Growth in Collapsed Liquid Crystal Monolayers Studied by Scanning Force Microscopy. Physica A, 244, 1-4, 91-98.

Feller, M.B., Chen, W. and Shen, Y.R. (1991) Investigation of Surface-Induced Alignment of Liquid-Crystal Molecules by Optical Second-Harmonic Generation. Phys. Rev. A, 43, 12, 6778-6792.

Frantz, P., Agrait, N. and Salmeron, M. (1996) Use of Capacitance to Measure Surface Forces. 1. Measuring Distance of Separation with Enhanced Spatial and Time Resolution. Langmuir, 12, 13, 3289-3294.

Fujihira, M. (1999) Kelvin Probe Force Microscopy of Molecular Surfaces. Ann. Rev. Mater. Sci., 29, 353-380.

Gao, C. and Xiang, X.D. (1998) Quantitative Microwave NearField Microscopy of Dielectric Properties. Rev. Sci. Instrum., 69, $11,3846-3851$.

Hao, H.W., Baro, A.M. and Saenz, J.J. (1991) Electrostatic and Contact Forces in Force Microscopy. J. Vac. Sci. Technol. B, 9, 2, 1323-1328.

Henning, A.K., Hochwitz, T., Slinkman, J., Never, J., Hoffmann, S., Kaszuba, P. and Daghlian, C. (1995) Two-Dimensional Surface Dopant Profiling in Silicon Using Scanning Kelvin Probe Microscopy. J. Appl. Phys., 77, 5, 1888-1896.

Hu, J., Xiao, X.D. and Salmeron, M. (1995a) Scanning Polarization Force Microscopy: A Technique for Imaging Liquids and Weakly Adsorbed Liquids. Appl. Phys. Lett., 67, 4, 476-478.

Hu, J., Xiao, X.D., Ogletree, D.F. and Salmeron, M. (1995b) The Structure of Molecularly Thin Films of Water on Mica in Humid Environments. Surf. Sci., 344, 3, 221-236.

Hu, J., Xiao, X.D., Ogletree, D.F. and Salmeron, M. (1995c) Imaging the Condensation and Evaporation of Molecularly Thin Films of Water with Nanometer Resolution. Science, 268, 5208, 267-269.

Israelachvili, J.N. (1985) Intermolecular and Surface Forces, Academic Press, New York.

Israelachvili, J.N. and Adams, G.E. (1976) Direct Measurement of Long Range Forces between Two Mica Surfaces in Aqueous $\mathrm{KNO}_{3}$ Solutions. Nature, 262, 5571, 774-776.

Israelachvili, J.N., Tandon, R.K. and White, L.R. (1979) Measurement of Forces between Two Mica Surfaces in Aqueous Poly(Ethylene Oxide) Solutions. Nature, 277, 5692, 120-121.

Luna, M., Rieutord, F., Melman, N.A., Dai, Q. and Salmeron, M. (1998) The Adsorption of Water on Alkali Halides Surfaces Studied by Scanning Polarization Force Microscopy. J. Phys. Chem. A, 102, 34, 6793-6800.

Martin, Y., Abraham, D.W. and Wickramasinghe, H.K. (1988) High-Resolution Capacitance Measurement and Potentiometry by Force Microscopy. Appl. Phys. Lett., 52, 13, 1103-1105.

Pershan, P.S. (1989) Liquid Crystal Surfaces. J. Phys. Colloq., C7, 1-20.

Pompe, T., Fery, A. and Herminghaus, S. (1998) Imaging Liquid Structures on Inhomogeneous Surfaces by Scanning Force Microscopy. Langmuir, 14, 10, 2585-2588.

Pompe, T., Fery, A. and Herminghaus, S. (1999) Measurement of Contact Line Tension by Analysis of the Three-Phase Boundary with Nanometer Resolution. J. Adhes. Sci. Technol., 13, 10, $1155-1164$. 
Rieutord, F. and Salmeron, M. (1998) Wetting Properties at the Submicrometer Scale: A Scanning Polarization Force Microscopy Study. J. Phys. Chem. B, 102, 20, 3941-3944.

Salmeron, M., Xu, L., Hu, J. and Dai, Q. (1997) High Resolution Imaging of Liquid Structures: Wetting and Capillary Phenomena at the Nanometer Scale. MRS Bull., 22, 8, 36-41.

Schick, M. (1990) Introduction to Wetting Phenomena, in Liquid at Interfaces, Charvolin, J., Joanny, J.F. and Zinn-Justin, J. (eds.), Elsevier Science Publishers, B.V., North-Holland, 415-498.

Schönenberger, C. and Alvarado, S.F. (1990) Observation of Single Charge Carriers by Force Microscopy. Phys. Rev. Lett., 65, 25, 3162-3164.

Sheiko, S., Lermann, E. and Möller, M. (1996) Self-Dewetting of Perfluoroalkyl Methacrylate Films on Glass. Langmuir, 12, 16, 4015-4024.

Sheiko, S.S., Muzafarov, A.M., Winkler, R.G., Getmanova, E.V., Eckert, G. and Reineker, P. (1997) Contact Angle Microscopy on a Carbosilane Dendrimer with Hydroxil End Groups: Method for Mesoscopic Characterization of the Surface Structure. Langmuir, 13, 15, 4172-4181.

Stevens, F., Patrick, D.L., Cee, V.J., Purcell, T.J. and Beebe, T.P., Jr. (1998) Transition from Epitaxial to Nonepitaxial Ordered Monolayers in Pyrolyzed 8CB Studied by STM. Langmuir, 14, 9, 2396-2401.

Takeuchi, I., Wei, T., Duewer, F., Yoo, Y.K., Xiang, X.D., Talyansky, V., Pai, S.P., Chen, G.J. and Venkatesan, T. (1997) Low Temperature Scanning-Tip Microwave Near-Field Microscopy of $\mathrm{YBa}_{2} \mathrm{Cu}_{3} \mathrm{O}_{7-\mathrm{x}}$ Films. Appl. Phys. Lett., 71, 14, 2026-2028.

Valignat, M.P., Fraysse, N., Cazabat, A.M., Heslot, F. and Levinson, P. (1993) An Ellipsometric Study of Layered Droplets. Thin Solid Films, 234, 1-2, 475-477.

Valignat, M.P., Villette, S., Li, J., Barberi, R., Bartolino, R., Dubois-Violette, E. and Cazabat, A.M. (1996) Wetting and
Anchoring of a Nematic Liquid Crystal on a Rough Surface. Phys. Rev. Lett., 77, 10, 1994-1997.

Van der Weide, D.W. (1997) Localized Picosecond Resolution with a Near-Field Microwave/Scanning- Force Microscope [IC Testing]. Appl. Phys. Lett., 70, 6, 677-679.

Villette, S., Valignat, M.P., Cazabat, A.M., Schabert, F.A. and Kalachev, A. (1997a) Ultrathin Liquid Films. Ellipsometric Study and AFM Preliminary Investigations. Physica A, 236, 1-2, 123129.

Villette, S., Valignat, M.P. and Cazabat, A.M. (1997b) Layered Spreading Microdroplets. J. Mol. Liq., 71, 2-3, 129-135.

Wei, T., Xiang, X.D., Wallace-Freedman, W.G. and Schultz, P.G. (1996) Scanning Tip Microwave Near-Field Microscope. Appl. Phys. Lett., 68, 24, 3506-3508.

$\mathrm{Xu}$, L. and Salmeron, M. (1998a) Scanning Polarization Force Microscopy Study of the Condensation and Wetting Properties of Glycerol on Mica. J. Phys. Chem. B, 102, 37, 7210-7215.

Xu, L. and Salmeron, M. (1998b) An XPS and Scanning Polarization Force Microscopy Study of the Exchange and Mobility of Surface Ions on Mica. Langmuir, 14, 20, 5841-5844.

Xu, L., Lio, A., Hu, J., Ogletree, D.F. and Salmeron, M. (1998) Wetting and Capillary Phenomena of Water on Mica. J. Phys. Chem. B, 102, 3, 540-548.

$\mathrm{Xu}$, L., Salmeron, M. and Bardon, S. (2000) Wetting and Molecular Orientation of 8CB on Silicon Substrates. Phys. Rev. Lett., 84, 7, 1519-1522.

Yokoyama, H., Jeffery, M.J. and Inoue, T. (1993) Heterodyne Force-Detection for High Frequency Local Dielectric Spectroscopy by Scanning Maxwell Stress Microscopy. Japanese Journal of Applied Physics, Part 2 (Letters), 32, 12B, L18451848.

Final manuscript received in September 2000 